\title{
MEAN CONVERGENCE OF WALSH FOURIER SERIES
}

\author{
CHINAMI WATARI
}

(Received March 6, 1964)

1. Let $\left\{\phi_{n}(x)\right\} n=1,2, \cdots$, be the system of Rademacher functions, i.e.

$$
\phi_{0}(x)=\left\{\begin{array}{r}
1(x \in[0,1 / 2)) \\
-1(x \in[1 / 2,1)),
\end{array} \quad \phi_{0}(x+1)=\phi_{0}(x), \phi_{n}(x)=\phi_{0}\left(2^{n} x\right),\right.
$$

and let

$$
\begin{aligned}
& \psi_{n}(x)=1 \quad \text { for } n=0, \text { and } \\
& \psi_{n}(x)=\phi_{n(1)}(x) \phi_{n(2)}(x) \cdots \phi_{n(r)}(x) \text { for } n=2^{n(1)}+2^{n(2)}+\cdots+2^{n(r)} \\
& \qquad n(1)>n(2)>\cdots>n(r) \geqq 0 .
\end{aligned}
$$

where

The functions thus defined are called the Walsh functions, which form a complete orthonormal system over the unit interval. We refer the reader to Paley [5] and Fine [1], for detailed properties of this system.

It was proved by Paley [5], that the Walsh Fourier series (abbrev. WFS) of a function $f(x)$ belonging to $L^{p}(0,1)(p>1)$ converges in $L^{p}$ norm to $f(x)$, but the corresponding results for functions in $L^{1}$ or $L^{1} \log ^{+} L^{1}$ have not yet been established, because the conjugate function, the most powerful tool in the theory of the trigonometric Fourier series, cannot be used in the theory of WFS.

The purpose of this note is to give a direct estimate for partial sums of the WFS of functions in $L^{1}$, and deduce the mean convergence of WFS in the so-called "critical" case. Let we write

$$
f(x) \sim \sum_{\nu=0}^{\infty} c_{\nu} \psi_{\nu}(x), c_{\nu}=\int f(t) \psi_{\nu}(t) d t
$$

the integral being taken over the unit interval and

$$
s_{n}(x)=s_{n}(x ; f)=\sum_{\nu=0}^{n-1} c_{\nu} \psi_{\nu}(x)=\int f(x+t) D_{n}(t) d t
$$

where + represents the "dyadic addition and 


$$
D_{n}(t)=\sum_{\nu=0}^{n-1} \psi_{\nu}(t)
$$

is the Dirichlet kernel for the Walsh system.

2. THEOREM 1. There exists an absolute constant $A>0$ such that for every $f \in L^{1}$, for every positive integer $n$ and for every positive number $y$, we have

$$
m\left(\left\{x ;\left|s_{n}(x ; f)\right|>y\right\}\right) \leqq A\|f\|_{1} / y .
$$

As the proof indicates, we may take $A=6$.

This theorem, combined with Bessel's inequality and the interpolation theorem of Marcinkiewicz ([7], vol. II, p. 112) and finally with a standard conjugacy argument, gives readily the following

THEOREM 2. (i) If $f \in L^{p}(1<p<\infty)$, then

$$
\left\|s_{n}(x ; f)\right\|_{p} \leqq A_{p}\|f\|_{p} .
$$

(ii) If $f \in L^{1} \log ^{+} L^{1}$, then

$$
\left\|s_{n}(x ; f)\right\|_{1} \leqq A \int|f(x)| \log ^{+}|f(x)| d x+A,
$$

where the constants $A_{p}, A$ depend neither on $f$ nor on $n$.

By a standard argument due to Kolmogoroff [4], we can see that Theorem 1 implies also

THEOREM 3. There exists a constant $A_{\mu}$, depending only on $\mu$, such that

$$
\left(\int\left|s_{n}(x ; f)\right| \not x\right)^{1 / \mu} \leqq A_{\mu}\|f\|_{1} \quad(0<\mu<1) .
$$

3. PROOF OF THEOREM 1. The proof proceeds in several steps.

LEMMA 1. Let $g \in L^{1}$ and let $y>\|g\|_{1}$ be a given number. Then we can decompose $g$ as follows:

$$
g=v+w, \quad w=\sum w_{i j} ;
$$

(ii) $\quad|v| \leqq 2 y$

for almost every $x$;

(iii) $\quad\|v\|_{1} \leqq\|g\|_{1}$; 
(iv) $\sum\left\|w_{i j}\right\|_{1} \leqq 4\|g\|_{1}$;

(v) there exists a countable system of disjoint "dyadic intervals" $I_{i j}$ :

$I_{i j}=I=\left[a, a+2^{-i}\right)$, where $a=a_{i j}$ is of the form $l / 2^{i}$ with $0 \leqq l<2^{i}$;

$\sum m\left(I_{i j}\right) \leqq\|g\|_{1} / y$, and $u=w_{i j}$ vanishes outside $I$;

(iv) $\int u d x=\int_{I} u d x=0$ for every pair $(i, j)$.

This Lemma is due to S. Igari and is a slight modification of the "covering lemma" of L. Hörmander. So we omit the proof, referring the reader to [2] or [3].

LeMma 2. Let $g \in L^{1}$ and let its WFS be $\sum b_{\nu} \psi_{\nu}(x)$. Write

$$
\delta_{0}(x ; g)=b_{0}, \delta_{k+1}(x ; g)=\sum\left\{b_{\nu} \psi_{\nu}(x) ; 2^{k} \leqq \nu<2^{k+1}\right\}
$$

Let $u=w_{i j}$ be a "piece" of $g$ in the previous lemma. Then

$$
\delta_{k}(x ; u)=0 \text { for } x \notin I=I_{i j} .
$$

PROOF. It is known that (cf. [1] and [5])

$$
\delta_{k+1}(x ; u)=\int u(t) \phi_{k}(x+t) D_{2^{k}}(x+t) d t
$$

and

$$
\delta_{k+1}(x+a ; u)=\int_{U} u(t+a) \phi_{k}(x+t) D_{2^{k}}(x+t) d t
$$

where $U=\left[0,2^{-i}\right)$, by (v) of Lemma 1 .

If $k<i$, we have $\phi_{\nu}(t)=1$ for $t \in U$ and $0 \leqq \nu \leqq k$, thus the Dirichlet kernel in the integrand is constant in the interval over which $u(t+a)$ has, by (vi) of Lemma 1 , the mean value 0 .

On the other hand, if $k \geqq i, x \notin I$ implies $D_{2^{k}}(x+t)=0$ for $t \in U$, since $(x+a \in I$ being equivalent to $x \in U) \quad x \notin U, t \in U$ imply $x$ 
$+t \notin U$ and $D_{2^{k}}(x+t)=D_{2^{k}}(x)=0$, for, as is well known,

$D_{2^{k}}(x)=\prod_{\nu=0}^{k-1}\left(1+\phi_{\nu}(x)\right)$.

As a corollary of Lemma 2, we have

LEMMA 3. $\delta_{k}(x ; w)=0 \quad$ for $\quad x \notin E=\bigcup I$.

PROOF. For $k=0$, the result follows from (vi) of Lemma 1. For other $k$ 's, it is sufficient to see that, by Lemma 2,

$$
\delta_{k}(x ; w)=\sum \delta_{k}(x ; u)=0 \quad \text { for } x \in C E=\bigcap C I .
$$

We can now complete the proof of Theorem 1. Let $f \in L^{1}$, a positive integer $n$ and $y>\|f\|_{1}$ be given. Put

$$
g(x)=f(x) \psi_{n}(x)
$$

Then, by an identity due to Paley ([5], p. 256)

$$
s_{n}(x ; f) \psi_{n}(x)=\delta_{n(1)}(x ; g)+\delta_{n(2)}(x ; g)+\cdots+\delta_{n(r)}(x ; g) .
$$

Decompose $g$ by Lemma 1 , obtaining

$$
s_{n}(x ; f) \psi_{n}(x)=\sum \delta_{n(k)}(x ; v)+\sum \delta_{n(k)}(x ; w)=V+W, \text { say. }
$$

$W$ vanishes outside $E$ by Lemma 3 ; thus

$$
\{x ;|W|>y\} \subset E
$$

and

$$
m(\{x ;|W|>y\}) \leqq\|g\|_{1} / y=\|f\|_{1} / y
$$

by Lemma 1, (v). On the other hand, Bessel's inequality gives

$$
\int|V|^{2} d x \leqq \int|v|^{2} d x \leqq 2 y \int|v| d x \leqq 2 y\|g\|_{1}=2 y\|f\|_{1}
$$

\section{Consequently}




$$
m(\{x ;|V|>y\}) \leqq y^{-2} \int|V|^{2} d x \leqq \frac{2}{y}\|f\|_{1} .
$$

Thus we obtain, for $y>\|f\|_{1}$,

$$
m\left(\left\{x ;\left|s_{n}(x ; f)\right|>2 y\right\}\right) \leqq 3\|f\|_{1} / y
$$

i. e.

$$
m\left(\left\{x ;\left|s_{n}(x ; f)\right|>y\right\} \leqq 6\|f\|_{1} / y \quad \text { for } y>2\|f\|_{1} .\right.
$$

But, for $y \leqq 2\|f\|_{1}$, it is clear that

$$
m\left(\left\{x ;\left|s_{n}(x ; f)\right|>y\right\}\right) \leqq 1 \leqq 2\|f\|_{1} / y,
$$

the proof is complete with $A=6$.

4. Lemma 3 also gives a proof of the following theorem due essentially to S. Yano [6].

Theorem 4. Put

$$
\delta^{*}(x ; f)=\sum_{k=0}^{\infty} \varepsilon_{k} \delta_{k}(x ; f) \quad \varepsilon_{k}=0,1 \text { or }-1
$$

Then

$$
m\left(\left\{x ;\left|\delta^{*}(x ; f)\right|>y\right\}\right) \leqq A\|f\|_{1} / y
$$

where $A$ is an absolute constant.

ProOF. Decompose $f$ itself by Lemma 1 . It is clear that

$$
\left|\delta^{*}(x ; f)\right| \leqq\left|\delta^{*}(x ; v)\right|+\left|\delta^{*}(x ; w)\right|
$$

and the second term on the right vanishes outside $E$ (Lemma 3). Párseval's equality will give the necessary estimate for $\left|\delta^{*}(x ; v)\right|$, and the rest of the proof may be left to the reader; a possible value of $A$ is 6 . 


\section{REFERENCES}

[1] N. J. FINE, On Walsh functions, Trans. Amer. Math. Soc., 65(1949), 372-414.

[2] L. HöRMANDER, Estimates for translation invariant operators in $L^{p}$ spaces, Acta Math., 104(1960), 93-140.

[ 3 ] S. IGARI, An extension of the interpolation theorem of Marcinkiewicz, Tôhoku Math. J., 15(1963), 343-358.

[4] A. KolmogorofF, Sur les functions harmoniques conjuguées et les séries de Fourier, Fund. Math., 7(1925), 24-29.

[5] R.E.A.C. PALEy, A remarkable system of orthogonal functions, Proc. London Math. Soc. 34(1932), 241-279.

[6] S. YANO, On a lemma of Marcinkiewicz and its applications to Fourier series, Tôhoku Math. J., 11(1959), 191-215.

[7] A.ZYGMUND, Trigonometric series, Cambridge, 1959.

TôHOKU UNIVERSITY 